\title{
Outcome of tibialis posterior tendon transfer to middle cuneiform by interference screw technique for foot drop
}

\author{
BLS Kumar Babu*, Biju Ravindran
}

Department of orthopaedics, Narayana Medical College, Nellore, Andhra Pradesh, India

Received: 21 August 2016

Accepted: 27 August 2016

\section{*Correspondence:}

Dr. BLS Kumar Babu,

E-mail: loknadh_bls@yahoo.co.in

Copyright: (C) the author(s), publisher and licensee Medip Academy. This is an open-access article distributed under the terms of the Creative Commons Attribution Non-Commercial License, which permits unrestricted non-commercial use, distribution, and reproduction in any medium, provided the original work is properly cited.

\section{ABSTRACT}

Background: Foot drop is a common problem which compromises the quality of life and impairs activities of daily living. Walking becomes difficult with foot drop because the foot quickly hits the obstacles, or the knee has to be more flexed than usual when going up stairs. Transfer of the tibialis posterior tendon restores active dorsiflexion of the foot, while tenodesis prevents the foot from dropping. To this purpose, we explored the results of this technique in a small cohort of foot drop patients.

Methods: Twenty-five patients with foot drop due to various causes such as tibial condyle fracture, fracture dislocation of the hip, post hip surgeries, late compartment syndrome and due to spinal aetiology by lumbar canal stenosis, post spinal surgeries due to discectomy and spondylolisthesis were included. Patients with post-traumatic arthritis of ankle, metabolic causes, poliomyelitis, sensory and motor neuropathy were excluded. Patients underwent anterior transposition of the tibialis posterior tendon (PTT) to the middle cuneiform through the interosseous membrane using tenodesis screw technique. The outcomes measured 2, 612 and 24 weeks postoperatively were a power of dorsiflexion, use of footwear to assess the dorsiflexion of the ankle in swing phase and at heel strike, hindfoot valgus patient satisfaction and activities of daily living.

Results: The average age of the patients was $46 \pm 7.4$ years. 18/25 (72\%) had the 4+/5 power of dorsiflexion, $7 / 25(28 \%)$ had $4-/ 5$ power. $23 / 25$ (92\%) of patients use of footwear of certain types, suggesting that these patients improved from a high-stepping gait to a heel-toe gait. 18/25 (72\%) patients were very satisfied, and 4/25 (16\%) were happy, $3 / 25(12 \%)$ patients were not satisfied. We found that $14 / 25(56 \%)$ were having regular daily activity, and rational recreation and none of our patient had a severe limitation on functional assessment. All the 25 patients in our cohort exhibited dorsiflexion of the ankle in swing phase by 12 weeks post operatively.3/25 (12\%) had hindfoot valgus postoperatively. None of our patients had wound complication at the insertion site.

Conclusions: Tenodesis screw technique for insertion site fixation showed $72 \%$ result in restoring ankle function with a similar patient satisfaction and minimal complications.

Keywords: Posterior tibialis transfer, Foot drop, Tenodesis

\section{INTRODUCTION}

Foot drop results from the loss of, ankle dorsiflexion from the tibialis anterior and foot eversion from the peroneus longus and brevis. ${ }^{1}$ Lesions of the peroneal nerve or damage to the tibialis anterior muscle are the primary causes of drop foot deformity. ${ }^{2}$ Walking becomes difficult with foot drop because the foot quickly hits the obstacles or the knee must be kept back more flexed than usual when going up stairs. Application of 
biomechanical and physiologic principles in tendon transfer procedures such as posterior tibialis tendon transfer, Bridle transfer, or variations on these methods can bring backdrop foot to near normalcy. ${ }^{3}$ The interosseous route has become the most accepted reconstructive method to correct drop- foot because it generates more direct pull, greater dorsiflexion power, less induction of unphysiological pronation and a better esthetic result. ${ }^{4}$ Tibialis posterior tendon transfer has assisted the gait and foot function in the long-term follow up. ${ }^{5}$ However, short tendon length and difficulties in securing the tendon to bone or tendon can complicate these procedures. ${ }^{6}$ A tenodesis if done proximal to the ankle joint can prevent these problems. Interference screw allows the surgeon to achieve the most favorable biomechanical tendon insertion producing efficient and balanced ankle dorsiflexion. Studies have shown that this technique restores ankle dorsiflexion with proper motor function. ${ }^{7,8}$ To this purpose, we also evaluated the results of this technique in a small cohort of foot drop patients.

\section{METHODS}

This study was conducted at Narayana Medical College Hospital during the years 2010-2015. Institutional ethics committee approved the study proposal. Both male and female patients between 20-60 years, having foot drop due to various causes such as tibial condyle fracture, fracture dislocation of hip, post hip surgeries, late compartment syndrome and due to spinal aetiology by lumbar canal stenosis, post spinal surgeries due to discectomy and spondylolisthesis are included. Patients with post-traumatic arthritis of ankle, metabolic causes, poliomyelitis, sensory and motor neuropathy were excluded. All these procedures were done by a single surgeon and tibialis posterior transfer to middle cuneiform tenodesis with interference screw technique was the surgery performed on all patients.

\section{Surgical procedure}

The harvesting incision was at the medial prominence of the navicular bone. After the tendon had been released, it was sutured with ethibond and sized for the appropriate tenodesis screw. A second incision was made at the posterior border of the tibia proximal to the medial malleolus, and the tendon was withdrawn through it. The index finger was passed along the posterior border of tibia through the second incision as blunt dissector to puncture the interosseous membrane and enter into the anterior compartment. The third incision was made over, to deliver the tendon anteriorly and care was taken to avoid twisting of the tendon. The final incision was made over middle cuneiform.

A subcutaneous tunnel was made with tendon passer and transferred tendon was drawn into it. The tendon was introduced into the middle cuneiform after drilling with an appropriate size cannulated drill chosen for tenodesis screw. Once the tendon was passed into the drill hole, the ankle was dorsiflexed in tension and tenodesis screw was secured into it.

\section{Follow-up}

The plaster cast kept for two months during which the patients had to walk with toe-touch weight bearing. Then the cast was removed and physiotherapy protocol initiated. The patient was evaluated regularly at 2, 612 and 24 weeks postoperatively.

The outcome was assessed based on five factors

1. Muscle power was judged by using modified Medical Research Council grading ranging from $4+$ or 5 to 2 or less. $^{9-10}$

2. Use of footwear- to assess patient's improvement from a high-stepping gait to a heel-toe gait.

3. Functional assessments ${ }^{11}$

- 4-Normal daily activity and normal recreation

- 3-Normal daily activity and limited recreation

- 2-Limited daily activity and recreation

- 1-Severe limitation on daily activity and recreation

4. Patients satisfaction on a10 point scale with $0-4=$ no satisfaction, 4-7=satisfied and >7 very much satisfied.

5. Post-operative hind foot valgus.

\section{Statistical analysis}

Data was entered into Excel spreadsheet 2007, cleaned and tabulated using pivotal tables. Continuous data was presented as straightforward arithmetic mean, category data as simple frequencies.

\section{RESULTS}

The causes for foot drop in ten patients were post spinal surgeries, in eight patients it was the post-traumatic origin, in five patients lumbar disc herniation and in remaining two patients post hip surgeries. The average age of the patients in our cohort was $46 \pm 7.4$ years. $18 / 25$ (72\%) had the $4+/ 5$ power of dorsiflexion, $7 / 25(28 \%)$ had $4-/ 5$ power. $23 / 25(92 \%)$ of patients use of footwear of certain types, suggesting that these patients improved from a high-stepping gait to a heel-toe gait. 18/25 (72\%) patients were very satisfied, and 4/25 (16\%) were happy, $3 / 25(12 \%)$ patients were not satisfied. We found that $14 / 25(56 \%)$ were having normal daily activity, and normal recreation and none of our patient had a severe limitation on functional assessment. All the 25 patients in our cohort exhibited dorsiflexion of the ankle in swing phase by 12 weeks postoperatively. 3/25 (12\%) had hindfoot valgus postoperatively. None of our patients had wound complication at the insertion site (Table 1). 
Table 1: Post-operative assessments.

\begin{tabular}{|lllll|}
\hline & $\mathbf{2}^{\text {nd }}$ week & $\mathbf{6}^{\text {th }}$ week & $\mathbf{1 2}$ weeks & 24 weeks \\
\hline Median muscle power & $4-/ 5$ & $4-/ 5$ & 4 plus & 4 plus \\
\hline Median functional assessment score & 2 & 2 & 3 & 3 \\
\hline $\begin{array}{l}\text { Percentage of patients who exhibiting } \\
\text { dorsiflexion of the ankle in swing phase }\end{array}$ & 0 & 0 & $100 \%$ & $100 \%$ \\
\hline Percentage of patients having hindfoot valgus & 0 & 0 & $3 / 25(12 \%)$ & $3 / 25(12 \%)$ \\
\hline
\end{tabular}

\section{DISCUSSION}

Drop foot has diverse etiologies among them traumatic peroneal nerve lesions are the most common cause. ${ }^{12}$ The objectives of posterior tibialis tendon transfer for treatment of food drop is to improve functional deficit by restoring or reinforcing lost functions; to neutralise deforming forces, and to gain stability eliminating the need for bracing during gait. ${ }^{13}$ These objectives can be accomplished by static and dynamic surgical procedures such as tendodesis and transferring functional muscles or changing their osseous insertions respectively. The tendon transfer is more efficient if dynamic; voluntary movement is achieved rather than just a static tenodesis effect whereas tenodesis effect is more likely in patients in older patients and in cases in which the surgical technique results in tendon adherence. ${ }^{14}$ Studies say that the tension between the tendon attachment into bone must be under slight to moderate tension transfer acts like a tenodesis than a dynamic transfer when the tension is greater than optimal. ${ }^{15,16}$ The novelty of our practice is that we bought the recipient tendon (ATT) right up to the PTT, resolving tendon harvest length problems and caring a straight line of pull. ${ }^{14}$

In our study, $72 \%$ of patients showed a $4+/ 5$ power of dorsi flexion postoperatively even by 12 weeks, $92 \%$ of patients' use of footwear of certain types, suggesting that these patients improved from a high-stepping gait to a heel-toe gait. $72 \%$ were highly satisfied. All the 25 patients in our cohort exhibited dorsiflexion of the ankle in swing phase by 12 weeks post operatively. Only $12 \%$ had hindfoot valgus postoperatively. Our experience did not produce any deficits regarding power and flexion of the ankle.

The possible mechanisms how tendon transfer works include the excursion of tibialis posterior tendon, being a unipennate muscle, is only $2 \mathrm{~cm}$ while that of tibialis anterior be- tween 3 to $5 \mathrm{~cm}$. As anticipated the passive arc of movement on the operated side is always less than the normal side, since the tendon fixed in dorsiflexion while the foot is usually in plantarflexion. ${ }^{17}$ The role of voluntary contraction of tibialis posterior is of greater importance than the tenodesis effect. The results are likely to be more successful if the nerve lesion is causing the drop- foot was at the common peroneal level rather than at the sciatic level and in men aged less than 30 years.

Caution has to be executed while interpreting our study results as we do not make any suggestions as to where the distal site of attachment should be but feel that this is likely to be largely determined by the existing muscular balance.

\section{CONCLUSION}

Tenodesis screw technique for insertion site fixation showed $72 \%$ result in restoring ankle function with a similar patient satisfaction and minimal complications and eliminates plantar pressure sores caused by fixation knot. Although passive plantar flexion is not complete after surgery, active plantar flexion at push-off was reduced to a greater extent.

\section{Funding: No funding sources}

Conflict of interest: None declared

Ethical approval: The study was approved by the institutional ethics committee

\section{REFERENCES}

1. Ho B, Khan Z, Switaj PJ, Ochenjele G, Fuchs D, Dahl W, et al. Treatment of peroneal nerve injuries with simultaneous tendon transfer and nerve exploration. J Orthop Surg Res. 2014;9(1):67.

2. Mehling I, Lanz U, Prommersberger KJ, Fuhrmann RA, van Schoonhoven J. Transfer of the posterior tibialis tendon to restore an active dorsiflexion of the foot. Handchirurgie, Mikrochirurgie, plastische Chirurgie: Organ der Deutschsprachigen Arbeitsgemeinschaft fur Handchirurgie : Organ der Deutschsprachigen Arbeitsgemeinschaft fur Mikrochirurgie der Peripheren Nerven und Gefasse 2012;44(1):29-34.

3. Schweitzer KM, Jones CP. Tendon transfers for the drop foot. Foot and ankle clinics. 2014;19(1):65-71.

4. Goh JC, Lee PY, Lee EH, Bose K. Biomechanical study on tibialis posterior tendon transfers. Clin Orthop Relat Res. 1995;319(319):297-302.

5. Partheebarajan S, Soloman S, Ebenezer M. Long term results of tibilalis posterior tendon transfer for 
foot drop in leprosy. Indian $\mathbf{J}$ Leprosy. 2012;84(2):145-9.

6. Wagenaar FC, Louwerens JW. Posterior tibial tendon transfer: results of fixation to the dorsiflexors proximal to the ankle joint. Foot \& ankle international. 2007;28(11):1128-42.

7. Jeng C, Myerson M. The uses of tendon transfers to correct paralytic deformity of the foot and ankle. Foot and ankle clinics. 2004;9(2):319-37.

8. Matuszak SA, Baker EA, Fortin PT. The adult paralytic foot. J American Academy of Orthopaedic Surgeons. 2013;21(5):276-85.

9. Van der Ploeg R, Oosterhuis H, Reuvekamp J. Measuring muscle strength. Journal of neurology. 1984;231(4):200-3.

10. Seddon HS. Surgical disorders of the peripheral nerves. $2^{\text {nd }}$ edition. Churchill Livingstone, Edinburgh: 1975.

11. Chua K, Kong K, Lui Y. Botulinum toxin A in the treatment of hemiplegic spastic foot drop-clinical and functional outcomes. Singapore Med J. 2000;41(5):209-13.

12. Vigasio A, Marcoccio I, Patelli A, Mattiuzzo V, Prestini G. New tendon transfer for correction of drop-foot in common peroneal nerve palsy. Clinical orthopaedics and related research. 2008;466(6):1454-66.

13. Mulier T, Moens P, Molenaers G, Spaepen D, Dereymaeker G, Fabry G. Split posterior tibial tendon transfer through the interosseus membrane in spastic equinovarus deformity. Foot \& ankle international. 1995;16(12):754-9.

14. Yeap JS, Birch R, Singh D. Long-term results of tibialis posterior tendon transfer for drop-foot. Int Orthop. 2001;25(2):114-8.

15. Hove LM, Nilsen PT. Posterior tibial tendon transfer for drop-foot. 20 cases followed for 1-5 years. Acta Orthop Scand. 1998;69(6):608-10.

16. Richard BM. Interosseous transfer of tibialis posterior for common peroneal nerve palsy. J Bone Joint Surg Br. 1989;71(5):834-7.

17. Marchi F, Malerba F, Montrasio Alfieri U, Ferrarin M, Rabuffetti M. Tibialis posterior tendon transfer through the interosseal membrane in paralysis of the common peroneal nerve. Foot and ankle surgery. 2000;6(1):19-25.

Cite this article as: Kumar Babu BLS, Ravindran B. Outcome of tibialis posterior tendon transfer to middle cuneiform by interference screw technique for foot drop. Int J Res Orthop 2016;2:177-80. 